\title{
Content validation of a tool to the Nursing Care Process for Pacients in Intensive Care Unit
}

Ráisa Camilo Ferreira (IC), Erika Christiane Marocco Duran (PQ)

\begin{abstract}
The intensivist nurse needs to carry out activities such as nurse team coordination and unit management, prescribing diagnosis of nursing care, using Nurse Process Method aiming to conduct the assistance and potentialise the treatment. The intention of this study is to elaborate and validate instrument to assist nursing, structured in checklist format, based on specific literature for intensive care patients. It is a developing research, occurring in three phases: Population analysis and verifying nursing diagnosis, instrument preparation and instrument validation (using Delphi Technique).
\end{abstract}

Key words: Patient Care Planning, Nursing Process, Intensive Care Units.

\section{Introduction}

Intensive care is an area of hospital assistance dedicated to critical patients' care, containing uninterrupted monitoring system with support and intensive care ${ }^{(1)}$.

The Intensivist nurse needs to be capable to carry out activities of great complex.

In this context, the Systematization of Nursing Care (SNC), defragments the care and organises the professional work, aiming to operationalise Nursing Process (NP) (1).

The construction of data collection instruments and printed containing the main diagnoses and nursing prescriptions appear in literature as methods to facilitate the implementation of NP and contribute to the quality of care ${ }^{(1)}$

\section{Results and Discussion}

During the data collection, 152 clients were hospitalised in Intensive Care, $61,18 \%$ were male $(\mathrm{N}=93)$, the average age was 54,9 . The average time of hospitalisation is 8,05 days. They were recorded among the study population, a rate of $13.16 \%$ deaths $(N=20)$. Of the total study population, $59.21 \%(\mathrm{~N}=90)$ showed risk factors and comorbidities, the most prevalent blood hypertension $30.05 \%(\mathrm{~N}=67)$, diabetes mellitus $20.18 \%(N=45)$ and dyslipidemia $10.31 \%(N=$ 23).

The medical diagnoses were heterogeneous, with a prevalence of heart disease $21 \%(N=32)$, sepsis / septic shock $19.13 \% \quad(\mathrm{~N}=29)$, multiple trauma and postoperative surgical $17.77 \%(\mathrm{~N}=27)$ each.
The nursing diagnoses (ND) more incidents were: Infection Risk $15.5 \%$, self-care deficit for bath $14.62 \%, 11.32 \%$ Impaired physical mobility, skin integrity Risk $11.15 \%$ and $10.50 \%$.

The population profile is consistent with that given in the literature, prevalence of male gender, age distribution in this study was is 50 to $6536.18 \%(\mathrm{~N}=55)$, and the average age 54.9 years was lower, than that found in previous studies ${ }^{(1)}$.

Among formulated ND, the risk of infection was a higher incidence, supporting the literature as well as the fact that in ICUs, patients exhibit a greater magnitude the actual ND and risk due to their clinical severity ${ }^{(1)}$.

\section{Conclusions}

It is concluded that the construction of data collection instruments and printed containing the main ND and nursing prescriptions are useful tools to facilitate the implementation of nursing care.

\section{Acknowledgement}

I would like to thank everyone who helped in the realization of this project, especially my supervisor Erika , my friends, family and jurors.

1.Zanotti R, Chiffi D. Diagnostic frameworks and nursing diagnoses: a normative stance. Nursing Philosophy (2015), 16, pp. 64-73. 